\title{
PCV Y PREGUNTAS MÚLTIPLES EN ESPAÑOL
}

\author{
Hui-Chuan Lu \\ National Cheng Kung University \\ hclu@mail.ncku.edu.tw
}

\section{RESUMEN}

El presente trabajo está dedicado a investigar diversas teorías que analizan las llamadas preguntas múltiples en español comparando y contrastando la extracción del sujeto, objeto y adjunto en inglés y en español, así como su relación con el Principio de Categoría Vacía (PCV) en el nivel de la Forma Lógica (FL). Llegaremos a la conclusión de que el hecho de que en español sea obligatoria la anteposición de V' en las preguntas de QU puede desempeñar un papel muy significativo para explicar el contraste entre el español y el inglés. Además, el problema provocado por la diferente aplicación del PCV en el nivel de la estructura superficial y en el de la Forma Lógica indica que el PCV debe ser modificado de alguna manera.

PALABRAS ClAVE: pregunta múltiple; Principio de Categoría Vacía; español; Forma Lógica.

\section{ABSTRACT}

In this paper, several different arguments with respect to multiple questions in Spanish will be discussed, by comparing and contrasting the extraction of subject, object and adjunct, as well as the application of ECP (Empty Category Principle) at LF (Logical Form) for the non-embedded multiple questions both in English and in Spanish. We conclude that Spanish obligatorily triggers $\mathrm{V}$-preposing in wh-question may play an important role in explaining the contrast between Spanish and English. Furthermore, the problems raised by applying ECP at S-structure and LF indicate that ECP must be modified in some ways in order to account for those problems raised during the discussion.

KeY WORDS: multiple question; Empty Category Principle; Spanish; Logical Form. 


\section{INTRODUCCIÓN}

En el presente trabajo, analizaremos en primer lugar algunas teorías diferentes que tratan sobre las llamadas preguntas múltiples en español, tales como las que observamos en los ejemplos (1) y (2) u oraciones con estructura similar. En la sección 3, compararemos y contrastaremos la extracción del sujeto, objeto y adjunto en inglés y en español; en el apartado 4, pondremos en relación las teorías estudiadas en las secciones anteriores con el Principio de Categoría Vacía (PCV), en el nivel de la Forma Lógica (FL) para las preguntas múltiples no-incrustadas en inglés y en español. Para terminar, en la sección 5, expondremos las conclusiones a las que hemos llegado en nuestro trabajo.

\section{ESTUDIOS PRECEDENTES}

Consideremos las oraciones (1) y (2) que se han estudiado en Contreras (1984) y que son similares a las estudiadas por Torrego (1984), Groos \& Bok-Kennema (1986) y Jaeggli (1988):

(1) ¿Qué paquete no sabes cuánto pesa?

Contreras (10a)

(2) * ¿Cuánto no sabes qué paquete pesa?

Contreras (10b)

Comparando dichos estudios, cabe distinguir al menos dos teorías diferentes: la de Contreras (1984) y la de Torrego (1984).

\subsection{Contreras (1984)}

Contreras (1984) cree que en español el orden de palabras dominante es VOS, y asigna a las oraciones (1) y (2) las siguientes estructuras respectivamente:

(3) [qué paquete ${ }_{\mathrm{i}}\left[\right.$ no sabes [cuánto ${ }_{\mathrm{j}}\left[\right.$ pesa $\left.\left.\left.\left.\mathrm{t}_{\mathrm{j}} \mathrm{t}_{\mathrm{i}}\right]\right]\right]\right]$

Contreras (11a)

(4) $*\left[\right.$ cuánto $_{\mathrm{j}}\left[\right.$ no sabes [qué paquete ${ }_{\mathrm{i}}\left[\right.$ pesa $\left.\left.\left.\left.\mathrm{t}_{\mathrm{j}} \mathrm{t}_{\mathrm{i}}\right]\right]\right]\right]$

Contreras (11b)

Además, el autor atribuye la agramaticalidad de la oración (2) al hecho de que la extracción de qué paquete y de cuánto viola la Restricción de Cruce. Asimismo, él explica que, si el español es una lengua de SVO, las oraciones (1) y (2) deben tener las siguientes estructuras superficiales:

(5) $\left[_{\mathrm{cp}}\right.$ qué paquete ${ }_{\mathrm{i}}\left[\mathrm{\text {nosabes }}\left[\mathrm{C}_{\mathrm{CP}}\right.\right.$ cuánto $_{\mathrm{j}}\left[_{\mathrm{IP}} \mathrm{t}_{\mathrm{i}}\right.$ pesa $\left.\left.\left.\left.\mathrm{t}_{\mathrm{j}}\right]\right]\right]\right]$

(6) $*\left[_{\text {cp }}\right.$ cuánto ${ }_{j}\left[\right.$ no sabes $\left[{ }_{C P}\right.$ qué paquete ${ }_{i}\left[{ }_{I P} t_{i}\right.$ pesa $\left.\left.\left.\left.t_{j}\right]\right]\right]\right]$ Contreras $(12 b)^{1}$

${ }^{1}$ M. Browning (1989) indica que hay tres versiones del Principio de Categoría Vacía (PCV):

(1) Chomsky (1981) sugiere —el PCV disyuntivo — en el que una categoría vacía está regida por una categoría de $\mathrm{X}^{\circ}$ (régimen léxico) o por una categoría coindexada (régimen de antecedente).

(2) En Chomsky (1986, sec. 11), el requisito del régimen apropiado del PCV se reduce a las categorías vacías nopronominales, que deben estar regidas por su antecedente. La asimetría de sujeto-objeto se correlaciona con la disponibilidad de las posiciones intermedias que permiten el régimen de antecedente.

(3) Jaeggli (1980), Chomsky (1986, sec. 12) y Stowell (1985) requieren ambos regímenes de antecedente y, además, alguna forma de cabeza/núcleo.

En el presente trabajo, el debate se basa en la versión (1) del PCV, el PCV disyuntivo. 
Por lo tanto, el PCV conducirá a una deducción falsa: en (5), el fragmento qué paquete $\left(t_{i}\right)$ no está regido léxicamente ni por ningún antecedente porque qué paquete aparece en la posición del Complemento (COMP) en cabeza, fuera de la proyección máxima que es el SC incrustado que contiene apropiadamente la traza $t_{i}$. Por consiguiente, ésta no está apropiadamente regida. Como el PCV está violado, se cree que la oración (1) debe ser agramatical. Sin embargo, esta conclusión es incorrecta. Por otro lado, en la oración (6), $t_{i}$ está regida por el antecedente qué paquete, que está en la posición del COMP incrustado, y la traza de cuánto, que es $t_{j}$, está regida léxicamente por el verbo pesa. Como ambas trazas están apropiadamente regidas, se deduce que la oración (2) es gramatical; no obstante, esta conclusión vuelve a ser incorrecta.

\subsection{Torrego (1984)}

Basándose en un orden de las palabras SVO y en la regla de anteposición de V que debe aplicarse a las preguntas de QU en las cláusulas incrustadas y en las no incrustadas, Torrego (1984) indica que las estructuras superficiales para las oraciones (7) y (8) son (9) y (10), respectivamente:

(7) ¿Quién no sabes cuánto pesa?

Torrego (40)

(8) *Cuánto no sabes quién pesa?

Torrego (43)

(9) $\left[_{c p}\right.$ quién $_{i}\left[{ }_{I P}\right.$ no sabes $\left[{ }_{C P}\right.$ cuánto $_{j}\left[\right.$ pesa $\left.\left.\left.\left._{v} t_{i} t_{v} t_{j}\right]\right]\right]\right]$

(10) $*\left[_{\text {cp }}\right.$ cuánto $_{j}\left[{ }_{I P}\right.$ no sabes $\left[{ }_{C P}\right.$ quién $_{i}\left[{ }_{I P}\right.$ pesa $\left.\left.\left.\left._{v} t_{i} t_{v} t_{j}\right]\right]\right]\right]$

La gramaticalidad de la oración (7) (con la estructura superficial (9)) se explica porque la traza de quién $\left(t_{i}\right)$ está regida léxicamente por el verbo pesa, y la traza de cuánto $\left(t_{j}\right)$ está regida por el antecedente cuánto, que está en la posición del COMP. Como ambas trazas $\left(t_{i} \mathrm{y} t_{j}\right)$ están apropiadamente regidas, no hay violación de PCV, y la oración (7) se interpreta, correctamente, como gramatical. Por otra parte, la agramaticalidad de la oración (8) se comprueba de igual modo: aunque la traza de quién $\left(t_{i}\right)$ está regida léxicamente (por pesa) y, al mismo tiempo, regida por el antecedente (quién en la posición del COMP incrustado), la traza de cuánto $(t)$ no está regida léxicamente porque la traza del verbo pesa $\left(t_{v}\right)$, como supone Torrego, no es un rector apropiado. Además, la traza $t_{j}$ no puede estar regida por el antecedente debido a que cuánto en el COMP encabezado no puede ser un antecedente local para la traza $t_{j}$ (cuánto está fuera de la proyección máxima: el SC incrustado que contiene $t_{j}$ ). Por consiguiente, la traza de cuánto no está regida apropiadamente y el PCV no se satisface; la oración (8) se interpreta, correctamente, como agramatical.

Tras examinar la discusión mencionada anteriormente, nos percatamos de que existen tres estructuras superficales propuestas para las oraciones $(1) /(2)$ (similares, tales como $(7) /(8))$ : (3)/(4), (5)/(6) y $(9) /(10)$.

Teniendo en cuenta lo propuesto por Contreras, el PCV indicará incorrectamente el resultado gramatical de las oraciones $(1) /(2)$ con las estructuras superficiales $(5) /(6)$; además, en las oraciones con las estructuras superficiales (3)/(4), repetidas abajo, el PCV también va a producir una conclusión incorrecta: $t_{i}$ no está regida léxicamente, ni regida 
por el antecedente en (3), de manera que la oración se trata incorrectamente como agramatical; por otra parte, ambas $t_{i}$ y $t_{j}$ están regidas apropiadamente en (4) y la oración sin duda se interpreta como gramatical:

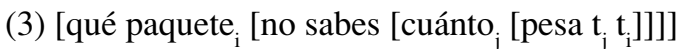

Contreras (11a)

(4) * cuánto $_{\mathrm{j}}\left[\right.$ no sabes [qué paquete ${ }_{\mathrm{i}}\left[\right.$ pesa $\left.\left.\left.\left._{\mathrm{j}} \mathrm{t}_{\mathrm{i}}\right]\right]\right]\right]$

Contreras (11b)

Por el momento, como las estructuras superficiales (3)/(4) y (5)/(6) plantean problemas a la aplicación del PCV, abandonaremos el análisis de Contreras y nos basaremos en el análisis de Torrego teniendo en cuenta para nuestro estudio, estructuras superficiales como las de (9)/(10).

\section{Extracción del Sujeto, Objeto y Adjunto}

\subsection{Extracción del Sujeto}

De la sección 2, en las oraciones (1) y (7) (¿Qué paquete no sabes cuánto pesa? y ¿Quién no sabes cuánto pesa?) la extracción del sujeto fuera de la pregunta incrustada nunca dirige a las oraciones inaceptables, al contrario de lo que se ocurre en inglés, donde las trazas de sujeto nunca están regidas léxicamente, por lo que tienen que cumplir el requisito de régimen de antecedente con el fin de satisfacer el PCV. Si los sujetos se extraen fuera de una isla de QU, la estructura se excluye porque la traza de sujeto (ej. $t_{i}$ en (11)) no es capaz de cumplir el régimen de antecedente:

(11) $*\left[_{c p}\right.$ Who $_{i}\left[{ }_{I P}\right.$ did you wonder $\left[{ }_{c p}\right.$ what $_{j}\left[{ }_{I P} t_{i}\right.$ bought $\left.\left.\left.\left.\left.t_{j}\right]\right]\right]\right]\right]$ Jaeggli (40)

Por otro lado, en español, la extracción del sujeto se realiza fuera de la posición postverbal debido a la regla de anteposición del verbo. Como se muestra en (13), el PCV explica correctamente la gramaticalidad de la oración $(12)^{2}$ por la misma razón mencionada para la estructura (9). Este hecho es completamente opuesto al inglés:

(12) ¿Quién no sabes qué compró? Jaeggli (45a)

(13) $\left[_{c p}\right.$ quién $_{i}\left[{ }_{I P}\right.$ no sabes $\left[_{c p}\right.$ qué ${ }_{j}\left[_{I P}\right.$ compró $\left.\left.\left.\left._{v} t_{i} t_{v} t_{j}\right]\right]\right]\right]$ Jaeggli (45b)

\subsection{Extracción del Objeto}

En inglés, la extracción del objeto no viola el PCV, ya que las formas de objeto pueden estar regidas léxicamente por los verbos, frente a lo que ocurre en español.

(14) *¿Qué no sabes quién compró?

(15) $\left[_{c p}\right.$ qué ${ }_{j}\left[\right.$ no sabes $\left[{ }_{c p}\right.$ quién ${ }_{i}\left[{ }_{I P}\right.$ compró $\left.\left.\left.\left._{v} t_{i} t_{v} t_{j}\right]\right]\right]\right]$

${ }^{2}$ En vez de usar los ejemplos de Torrego (7) y (8), propongo el ejemplo (12) para la siguiente discusión con el objetivo de señalar la extracción de sujeto y de objeto, porque no creo que cuánto en (7)/(8) deba considerarse como el complemento (u objeto) del verbo pesa. 
En inglés, a pesar de que la extracción del sujeto conduce a la violación del PCV, la extracción de objeto no da ese resultado. El contraste entre la extracción del sujeto y del objeto refleja una asimetría en el régimen del sujeto y del objeto: un verbo rige al objeto léxicamente, mientras que un sujeto nunca es regido léxicamente por un verbo.

Por otra parte, en español, la extracción del objeto producirá una oración agramatical como la del ejemplo (14); sin embargo, la extracción del sujeto, según muestran las oraciones de (1), (7) o (12), nunca determinará agramaticalidad. Es presumible que este contraste entre la posibilidad de extracción del sujeto y la del objeto también refleje una asimetría en el régimen de sujetos: en español, el verbo regirá léxicamente el elemento de su lado derecho (la posición de sujeto) cuando el verbo se sitúe al lado izquierdo del sujeto, tal como se muestra en (15): la posición del objeto no puede estar regida léxicamente por el verbo compró, pero sí por la traza del verbo $t_{v}$, que no es un rector apropiado. Podemos observar que los elementos que se subcategorizan por los verbos no pueden mudarse fuera de las preguntas de $\mathrm{QU}$ en español porque a sus trazas les falta el régimen.

Del contraste expuesto anteriormente, el resultado opuesto que advertimos acerca de la asimetría del sujeto y del objeto en inglés y español puede deberse a que en español se requiere obligatoriamente la aplicación de la regla de la anteposición del verbo en las preguntas de QU, mientras que tal requisito no es obligatorio en inglés.

\subsection{Extracción del Adjunto}

Respecto a las oraciones con la extracción de adjunto fuera de las preguntas incrustados, tales como las de los ejemplos (16)-(21), vemos cómo se excluyen no sólo en inglés, sino también en español ${ }^{3}$ :

(16) *Where did John wonder what Mary bought?

(17) *Why did Mary wonder who John killed?

(18) *Why did you know when John left?

(19) * ¿Dónde no sabes qué compró María?

(20) *iPor qué no sabes a quién despidió Juan?

(21) * ¿Por qué no sabes cuánto salió Juan?

El adjunto no es un complemento de categoría alguna, de ahí que no pueda estar regido léxicamente por nada. Ello explica el hecho de que las trazas de adjunto tengan que cumplir el régimen de antecedente para satisfacer el PCV. La extracción del adjunto cruzando la isla de QU provocará un resultado inaceptable debido a que la traza de adjunto $\left(t_{k}\right)$ falla en el régimen de antecedente por palabra ${ }_{k}$ de QU en la posición del COMP situado en cabeza, como se muestra en las series (22)-(27), correspondientes a las estructuras superficiales de (16)-(21):

(22) $*\left[\right.$ where $_{\mathrm{k}}$ [did John wonder [what $\left[\right.$ [Mary bought $\left.\left.\left.\left.\mathrm{t}_{\mathrm{j}} \mathrm{t}_{\mathrm{k}}\right]\right]\right]\right]$

(23) $*\left[w_{\mathrm{k}} \mathrm{k}_{\mathrm{k}}\right.$ [did Mary wonder [who $\mathrm{w}_{\mathrm{j}}$ [John killed $\left.\left.\left.\left.\mathrm{t}_{\mathrm{j}} \mathrm{t}_{\mathrm{k}}\right]\right]\right]\right]$

\footnotetext{
${ }^{3}$ Las oraciones (16)-(21) son agramaticales cuando el adjunto en el COMP encabezado se interpreta con un alcance estrecho.
} 


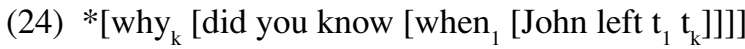

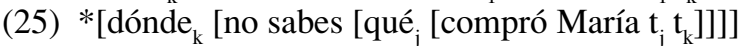

(26) $*\left[\right.$ por qué ${ }_{\mathrm{k}}$ [no sabes [a quién ${ }_{\mathrm{j}}$ [despidió Juan $\mathrm{t}_{\mathrm{j}} \mathrm{t}_{\mathrm{k}}$ ]]]]

(27) $*\left[\right.$ por qué ${ }_{\mathrm{k}}$ [no sabes [cuándo ${ }_{1}\left[\right.$ salió Juan $\left.\mathrm{t}_{1} \mathrm{t}_{\mathrm{k}}\right]$ ]]]

Respecto al inglés, Huang (1982) niega que exista asimetría de sujeto-objeto, y sostiene que la que existe es de complemento-no complemento, porque el sujeto (véase sección 2.1.) y el adjunto (véase 2.3.) no son complementos y, por lo tanto, no pueden estar regidos léxicamente. Tan pronto como su régimen de antecedente falla, no pueden satisfacer el PCV. La conclusión es, por consiguiente, que los no-complementos deben estar regidos por el antecedente en inglés, tal como propone Lasnik (1988).

Sin embargo, la asimetría de complemento-no complemento propuesta por Huang no puede aplicarse al caso del español, o a lenguas que obligatoriamente requieran la anteposición del verbo, porque el PCV se violará cuando el objeto o el adjunto se extraigan fuera de las frases de QU en español, mientras que el PCV no se viola si se extrae el sujeto. Por esta razón, en español, a diferencia del inglés, no hay una asimetría de complemento-no complemento, sino sólo asimetría sujeto-objeto/adjunto (no complementocomplemento/no complemento).

Podemos concluir hasta el momento que tanto el español como el inglés funcionan de manera similar con respecto a la extracción de un adjunto: la traza de un adjunto, en español y en inglés, puede estar regida sólo cuando su antecedente está en la posición del COMP incrustado. Por otro lado, el español y el inglés tienen patrones diferentes en cuanto a la extracción de sujeto-objeto.

\section{Problemas}

\subsection{Problemas con las reglas de la anteposición del verbo}

Siguiendo lo discutido en la sección 2.2., la extracción del objeto fuera de la frase de QU causará la violación del PCV; entonces, la oración (28) se va a considerar, de manera incorrecta, como una oración agramatical porque el objeto qué se muda cruzando el elemento de QU por qué.

(28) ¿Qué no sabes por qué compraste?

Torrego (1984) supone que existen ciertas palabras QU (incluye por qué, cuándo, cómo, en qué medida) que no requieren obligatoriamente la anteposición de verbo. Si seguimos el análisis propuesto por Torrego, en la estructura (29) (estructura superficial de la oración (28)), el movimiento de QU puede libremente extraer un objeto subcategorizado por un verbo desde una pregunta de QU porque el verbo de la pregunta incrustada, compró, no se antepone y todavía puede regir léxicamente la traza del objeto, $t_{j}$; por lo tanto, a diferencia de lo ocurrido con la oración (14), el PCV no se violará en la oración (28), aunque el objeto se extraiga fuera de la frase de QU. De este modo, la conclusión de Torrego (1984) es que el elemento mudado por el movimiento de QU desde las preguntas incrustadas va a producir una oración inaceptable porque implica el movimiento de 
una categoría contenida en el sintagma verbal desde una pregunta que requiere la anteposición del verbo, como ocurre, por ejemplo, en (14), pero no (28).

(29a) [qué ${ }_{j}\left[\right.$ no sabes [por qué ${ }_{k}\left[(\right.$ pro $)$ compraste $\left.\left.\left.\left.t_{j} t_{k}\right]\right]\right]\right]$

(29b) [qué ${ }_{j}$ [no sabes [por qué ${ }_{k}$ [compraste (pro) $\mathrm{t}_{\mathrm{j}} \mathrm{t}_{\mathrm{k}}$ ]]]]

En (29), el sujeto de la cláusula incrustada es un elemento nulo, pro, cuya posición no se puede examinar para ver si se sitúa antes o después del verbo compraste: (29a) o (29b). Sin embargo, propondré lo mismo que argumenta Torrego: por qué en el COMP de una pregunta no-incrustada no obliga a la aplicación de la regla de anteposición del verbo, como se muestra en (30) y (31) (en (31), por qué precede a un sujeto preverbal), mientras que por qué en la posición del COMP incrustado requiere obligatoriamente la aplicación de la regla de anteposición del verbo, como se señala en (32) y (33):

(30) ¿Por qué María salió?

(31) ¿Por qué salió María?

(32) ¿Qué no sabes por qué compró María?

(33) *QQué no sabes por qué María compró?

Además, consideramos qué estructura superficial, (34) o (35), debe asignarse a la oración (32):

(34) [qué [no sabes [por qué ${ }_{\mathrm{k}}$ [compró ${ }_{\mathrm{v}}$ María $_{\mathrm{v}} \mathrm{t}_{\mathrm{j}} \mathrm{t}_{\mathrm{k}}$ ]] ]

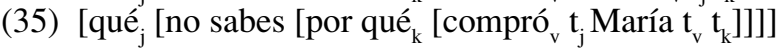

Hasta ahora, hemos tenido en cuenta construcciones similares a (34) como estructura superficial válida para estos casos. No obstante, si con la anteposición de verbo (así llamada por Torrego) nos referimos a la anteposición de $\mathrm{V}^{\circ}$, la oración (32) con la estructura superficial (34) va a interpretarse, de manera errónea, como agramatical porque la traza del objeto $t_{j}$ no puede estar regida apropiadamente; por el contrario, si la anteposición de verbo se refiere a la anteposición de V', la estructura superficial para (32) va a ser (35) en vez de (34): en (35), como $t_{j}$ está léxicamente regida por el verbo compró y, además, $t_{k}$ está regida por su antecedente local por qué, el PCV no se viola y la oración (32) se predice, de manera acertada, como gramatical, cuando se interpreta bajo dicha estructura superficial.

De todo lo visto anteriormente, podemos concluir, en primer lugar, que una palabra QU como por qué requiere obligatoriamente la anteposición de un verbo a la pregunta incrustada antepuesta, como en (32); si bien no es necesaria en las preguntas no-incrustadas, tal como ocurre en (30)/(31). En segundo lugar, si la anteposición de verbo (como propone Torrego) no es la anteposición de $\mathrm{V}^{\circ}$, sino la anteposición de $\mathrm{V}^{\prime}$, resulta la estructura superficial (35), lo que contradice lo discutido en las secciones anteriores: es decir, la estructura superficial para (7) y (8) han de ser (36) y (37), y no (9) y (10):

(36) [quién ${ }_{i}$ [no sabes [qué [compró $\mathrm{t}_{\mathrm{j}} \mathrm{t}_{\mathrm{i}} \mathrm{t}_{\mathrm{v}}$ ]]]]

(37) *[qué ${ }_{j}\left[\right.$ no sabes [quién ${ }_{\mathrm{i}}\left[\right.$ compró $\left.\left.\left._{\mathrm{v}} \mathrm{t}_{\mathrm{j}} \mathrm{t}_{\mathrm{i}} \mathrm{t}_{\mathrm{v}}\right]\right]\right]$ ]

Entonces, el PCV deducirá, incorrectamente, que (36) es agramatical: $t_{j}$ está regida, 
léxicamente, por compró y por el antecedente qué; en cambio, $\mathrm{t}_{\mathrm{i}}$ no está regida ni, léxicamente, por el verbo ni por el antecedente, por lo que el PCV se viola. Y el PCV también va a interpretar, incorrectamente, que (37) es gramatical: como $t_{j}$ está regida léxicamente por compró y $t_{i}$ está regida por el antecedente quién, no hay violación del PCV. De esta manera parece que tendremos una cuarta estructura superficial para las oraciones (7)/(8) (o estructuras similares, como (1) y (2)), pero esta cuarta estructura superficial, como hemos visto, causa más problemas que los que resuelve. Sugiero que consideremos (32) como un problema, y reservemos la regla de anteposición de $\mathrm{V}$ sólo para la de $\mathrm{V}^{\circ}$ y no para la de $\mathrm{V}^{\prime}$.

\subsection{Problema con el PCV en Forma Lógica}

Hasta este punto, lo que hemos considerado es la aplicación del PCV en el nivel sintáctico para las preguntas incrustadas. Hemos supuesto que el PCV tiene lugar en el nivel de FL en vez de en la estructura superficial (Kayne 1981). En las preguntas múltiples noincrustadas en inglés, el PCV predice, correctamente, el resultado de las siguientes oraciones (38), (40), (42), (44), (46) y (48):

(38) Who saw what?

Siguiendo las hipótesis de la indexación propuestas por Lasnik (1988), la representación de FL para (38) será (39): Who se ha mudado en el nivel sintáctico y, por lo tanto, es la cabeza del COMP, y se queda allí cuando what adjunta al COMP mediante el movimiento de QU en el nivel de FL:

$$
\left.\mathrm{C}_{\text {Comp }} \text { what }_{\mathrm{j}}\left[_{\mathrm{Comp}} \mathrm{Who}_{\mathrm{i}}\right]_{\mathrm{i}}\right]_{\mathrm{i}}\left[\mathrm{t}_{\mathrm{i}} \text { saw } \mathrm{t}_{\mathrm{j}}\right]
$$

En (39), $\mathrm{t}_{\mathrm{i}}$ está regida por el antecedente who y $t_{j}$ está léxicamente regida por saw. Como ambas trazas están apropiadamente regidas, no existe violación del PCV, y la oración (38) se interpreta, de forma correcta, como gramatical.

(40) *What did who see?

(41) Representación de FL: $\left.\left[\text { Comp }_{\text {who }}\left[_{\text {Comp }} \text { what }\right]_{j}\right]_{j}\right]_{j}\left[\right.$ did $t_{i}$ see $\left.\left.\left.t_{j}\right]\right]\right]$

En (41), aunque $t_{j}$ está léxicamente regida por see y regida por el antecedente Comp what, $t_{i}$ no está apropiadamente regida por nada: ni está léxicamente regida por el verbo ni por el antecedente; por lo tanto, el PCV se viola y la oración se predice, de manera acertada, como agramatical.

(42) *Who left why?

(43) Representación de FL: $\left[_{\text {Comp }} \text { why }_{\mathrm{k}}\left[_{\text {Comp }} \text { who }_{\mathrm{i}}\right]_{\mathrm{i}}\right]_{\mathrm{i}}\left[\mathrm{t}_{\mathrm{i}}\right.$ left $\left.\mathrm{t}_{\mathrm{k}}\right]$

Si observamos (43), $t_{i}$ está regida por el antecedente $w h o$, pero $t_{k}$ no está apropiadamente regida porque, al ser un adjunto, no puede estar léxicamente regida ni regida por el antecedente, por lo que el PCV se viola y la oración se predice, correctamente, agramatical.

(44) *Why did who leave? 
(45) Representación de FL: $\left[w o_{k}\left[w_{h}\right]_{k}\right]_{k}\left[\right.$ did $t_{i}$ leave $\left.t_{k}\right]$

En el caso de (45), aunque $t_{k}$ esté apropiadamente regida (regida por el antecedente), $t_{i}$ no lo está, y la oración debe considerarse, correctamente, como agramatical.

(46) *What did you buy why?

(47) Representación de FL: $\left[_{\text {Comp }} \text { why }{ }_{k}\left[{ }_{\text {Comp }} \text { what }_{j}\right]_{j}\right]_{j}$ [did you buy $t_{j} t_{k}$ ]

Teniendo en cuenta la propuesta (47), $t_{j}$ está léxicamente regida por buy y, al mismo tiempo, regida por el antecedente what, pero $t_{k}$ no puede estar apropiadamente regida por la misma razón que $t_{k}$ en (43), por eso, la oración se predice, correctamente, agramatical.

(48) Why did you buy what?

(49) Representación de FL: [ what $\left.{ }_{j}\left[\text { why }_{\mathrm{k}}\right]_{\mathrm{k}}\right]_{\mathrm{k}}$ [did you buy $\mathrm{t}_{\mathrm{j}} \mathrm{t}_{\mathrm{k}}$ ]

Si analizamos (49), como $t_{j}$ está léxicamente regida por buy y $t_{k}$, por el antecedente why, la oración se predice, acertadamente, gramatical. Por otra parte, el PCV no puede predecir el resultado correcto de la gramaticalidad para las oraciones paralelas en español (50), (54), (56) y (58):

(50) ¿Quién vio a quién?

(51) Representación de FL: [a quién $\left.{ }_{\mathrm{j}}\left[\text { quién }_{\mathrm{i}}\right]_{\mathrm{i}}\right]_{\mathrm{i}}\left[\mathrm{vio}_{\mathrm{v}} \mathrm{t}_{\mathrm{i}} \mathrm{t}_{\mathrm{v}} \mathrm{t}_{\mathrm{j}}\right.$ ]

En (51), $t_{i}$ está regida, léxicamente, por vio y por el antecedente quién, pero $t_{j}$ no está apropiadamente regida, y la oración se predice, incorrectamente, agramatical.

(52) ¿A quién vio quién?

(53) Representación de FL: [ quién $\left.n_{i}\left[a \text { quién }_{j}\right]_{j}\right]_{j}\left[\operatorname{vio}_{v} t_{i} t_{v} t_{j}\right]$

En (53) $t_{i}$ está regida, léxicamente, por vio y $t_{j}$ y por el antecedente quién, por lo que la oración se considera, correctamente, como gramatical.

(54) ¿Quién salió por qué?

(55) Representación de FL: [por qué $\left.{ }_{\mathrm{k}}\left[\text { quién }_{\mathrm{i}}\right]_{\mathrm{i}}\right]_{\mathrm{i}}$ [salió $\mathrm{t}_{\mathrm{i}} \mathrm{t}_{\mathrm{v}} \mathrm{t}_{\mathrm{k}}$ ]

Respecto a (55), $t_{i}$ está regida por el antecedente quién, pero $t_{k}$ no se rige adecuadamente, por lo que la oración se predice, de manera incorrecta, como agramatical.

(56) ¿Por qué salió quién?

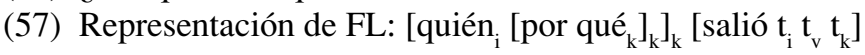

En el caso de (57), $t_{k}$ está regida por el antecedente por qué, pero $t_{i}$ no puede estar apropiadamente regida, por lo que se predice, incorrectamente, como agramatical.

(58) ¿Qué compraste por qué?

(59) Representación de FL: [por qué $\left.{ }_{k}\left[q u e_{j}\right]_{j}\right]_{j}\left[\right.$ compraste $t_{j} t_{k}$ ]

En (59), $t_{j}$ está léxicamente regida por compraste, pero $t_{k}$ no está apropiadamente regida; por lo tanto, se considerará como una oración agramatical, aun cuando la predicción sea incorrecta. 
(60) ¿Por qué compraste qué?

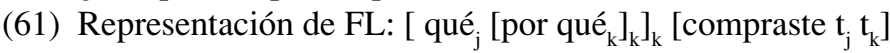

Por último, en (61), $t_{j}$ está léxicamente regida por compraste, y, además, $t_{k}$ está regida por el antecedente por qué, por lo que la oración se predice, de manera acertada, como gramatical.

Así las cosas, cabe hacer la siguiente observación: en español, el PCV no funciona en el nivel de FL, frente a lo que ocurre en inglés.

\section{CONCLUSIÓN}

Después de analizar la extracción del sujeto, el objeto y el adjunto en inglés y español, y tratar los «problemas» derivados de las hipótesis anteriores sobre la aplicación del $\mathrm{PCV}$, concluimos que el hecho de que el español obligue a la anteposición de V' en las preguntas de QU puede desempeñar un papel muy importante para explicar el contraste entre el español y el inglés. El problema provocado por la diferencia en la aplicación del PCV en el nivel de la estructura superficial y en el de la Forma Lógica nos está indicando que el PCV debe ser modificado de alguna manera. Tenemos confianza en que pronto encontraremos la clave para resolver los diversos problemas suscitados en el presente trabajo.

\section{REFERENCIAS BIBLIOGRÁFICAS}

BRownING, Marguerite (1989): «PCV $\neq$ CED», LI 20, págs. 481-491.

CHомsкy, Noam (1981): Lectures on Government and Binding, Dorcrecht, Foris, págs. 248-252.

- (1986): Barriers, Cambridge, Mass., MIT Press.

CONTRERAS, Heles (1984): «Multiple questions in English and Spanish», Papers from the XII the Linguistic Symposium on Romance Languages, págs. 121-133.

Groos, Anneke y BoK-BEnNEMA, Reineke (1986): «The structure of the sentence in Spanish», Generative Studies in Spanish Syntax, págs. 67-80.

JAEGGLI, Osvaldo (1980): Topics in Romance Syntax, Dordrecht: Foris.

- (1988): «PCV effects at FL in Spanish», Advances in Romance Linguistics, págs. 113-149.

KAYNE, Richard (1981): «PCV extensions», LI 12, págs. 93-133.

LASNIK, Howard y URIAGEREKA, Juan (1988): A Course in GB Syntax: Lectures on Binging and Empty Categories, Cambridge, Mass., MIT Press, págs. 92-116.

STOWELL, Tim (1985): «Null operators and the theory of proper government», ms., UCLA, Los Ángeles, California.

TORREGO, Esther (1984): «On inversion in Spanish and some of its effects», LI 15, págs. 103-129. 